\title{
Beta-function for the Higgs self-interaction in the Standard Model at three-loop level
}

\author{
Max ZOLLER* \\ Karlsruhe Institute of Technology \\ E-mail: max.zoller@kit.edu
}

\begin{abstract}
The discovery of a Higgs particle [1,2] has triggered numerous theoretical and experimental investigations concerning its production and decay rates and has led to interesting results concerning its interaction with fermions and gauge bosons. The self-interaction $\lambda$ of the Standard Model Higgs boson is particularly important due to its close connection with the stability of the SM vacuum. In this talk precision calculations for the evolution of this crucial coupling are presented and their impact on the question of vacuum stability is analysed. We also compare the theoretical precision resulting from the calculation of three-loop $\beta$-functions to the experimental uncertainties stemming from key parameters, such as the top mass, the Higgs mass and the strong coupling, and to the theoretical uncertainties introduced by the matching of experimental data to parameters in the theoretically favoured $\overline{\mathrm{MS}}$ renormalization scheme.
\end{abstract}

The European Physical Society Conference on High Energy Physics

18-24 July, 2013

Stockholm, Sweden

* Speaker. 


\section{Introduction}

The Standard Model (SM) of particle physics is an $S U(3) \times S U(2) \times U(1)$ gauge theory describing the interactions of fermions through the exchange of gauge bosons. In addition a scalar $S U(2)$ doublet is introduced which aquires a vacuum expectation value (VEV) at the electroweak scale and produces the Higgs field and three Goldstone bosons. The fermion masses and the Higgsfermion interaction are described by the Yukawa sector of the SM and the Higgs self-interaction is introduced to the Lagrangian in the Higgs potential which classically reads

$$
V(\Phi)=\left(m^{2} \Phi^{\dagger} \Phi+\lambda\left(\Phi^{\dagger} \Phi\right)^{2}\right), \quad \Phi=\left(\begin{array}{c}
\Phi_{1} \\
\Phi_{2}
\end{array}\right) \stackrel{\operatorname{SSB}}{\longrightarrow}\left(\begin{array}{c}
\Phi^{+} \\
\frac{1}{\sqrt{2}}(v+H-i \chi)
\end{array}\right) .
$$

The strength of each interaction is given by a coupling constant (see Fig. 1). ${ }^{1}$

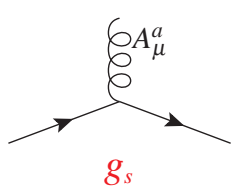

$[S U(3)]$

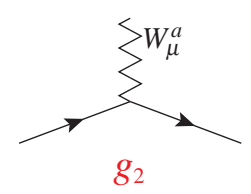

$[S U(2)]$

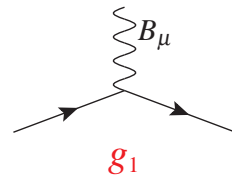

$[U(1)]$

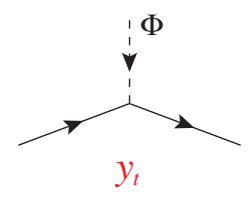

[Yukawa (top)]

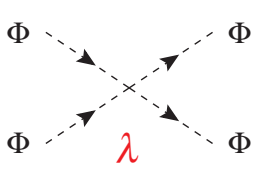

[Higgs self-coupling]

Figure 1: SM interactions

In the absence of physics beyond the SM at the LHC so far it is conceivable to extrapolate the SM to high energies, eventually even up to the Planck scale. A problem which arises as a consequence of radiative corrections is the possibility of a second minimum in the effective Higgs potential, lower than the one at the electroweak scale, which would result in an unstable or metastable electroweak vacuum state.

The effective potential [3] is affected by the self-interactions of the scalar fields as well as the interactions of the scalar fields with all other fields. Hence it depends on all the SM couplings evolved from some initial scale, e.g. the top pole mass $M_{t}$, up to the upper limit $\Lambda$ of the validity of the theory, e.g. $\Lambda=M_{\text {Planck }}$.

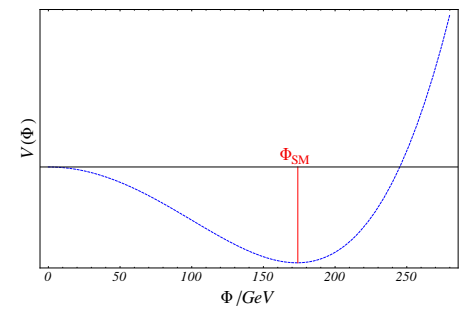

classical situation (tree level)

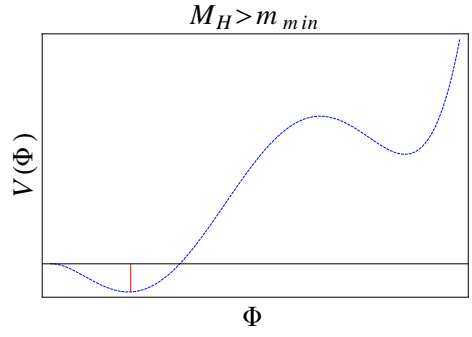

$$
\frac{\text { stable }}{\lambda(\mu)>0 \forall \mu \leq \Lambda}
$$

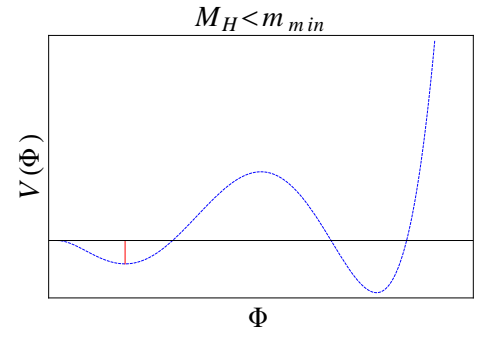

unstable/metastable $\exists \mu \leq \Lambda: \lambda(\mu)<0$

Figure 2: Classical and effective Higgs potential

\footnotetext{
${ }^{1}$ Except for $y_{t}$ the Yukawa interactions $y_{b}, y_{c}, \ldots$ can be neglected due to their smallness. The same applies to the off-diagonal entries of the CKM-matrix.
} 
Generic shapes of the classical Higgs potential and of the effective potential are shown in Fig. 2 for the cases of a Higgs mass larger and smaller than a critical value $m_{\min }$, the minimal stability bound $^{2}$ (see also [4]). It has been demonstrated that the stability of the SM vacuum is in good approximation equivalent to the question whether $\lambda$ stays positive up to the scale $\Lambda$ [5-7]. For a detailed discussion of the vacuum stability problem in the SM see $[4,8-14]$.

\section{The three-loop $\beta$-function for the Higgs self-interaction}

The evolution of the Higgs self-coupling $\lambda$ is described by the $\beta$-function

$$
\beta_{\lambda}\left(\lambda, y_{t}, g_{s}, g_{2}, g_{1}, \ldots\right)=\mu^{2} \frac{d}{d \mu^{2}} \lambda(\mu)
$$

which is a power series in all couplings of the SM and has recently been computed at three-loop level $[12,15,16]$. During the last two years the $\beta$-functions for the gauge [17-19] and Yukawa [12, 20] couplings have been calculated at three-loop level as well. In order to determine the evolution of $\lambda$ we need to solve the coupled system of differential equations

$$
\beta_{X}\left(\lambda, y_{t}, g_{s}, g_{2}, g_{1}\right)=\mu^{2} \frac{d}{d \mu^{2}} X(\mu), \quad X \in\left\{\lambda, y_{t}, g_{s}, g_{2}, g_{1}\right\} .
$$

Furthermore, in order to solve eq. (2.2) an initial condition for each coupling has to be given. One possible choice is to take their values at the scale of the top mass $M_{t}$. As the $\beta$-functions have been calculated in the $\overline{\mathrm{MS}}$-scheme but parameters like the top and Higgs mass are (in good approximation) determined on-shell by experiments ${ }^{3}$, we have to use matching relations between on-shell and $\overline{\mathrm{MS}}$ parameters at two-loop level [4, 8, 42-45]. For the pole masses $M_{t}=173.07 \mathrm{GeV}$ and $M_{H}=125.9 \mathrm{GeV}$ and $\alpha_{s}\left(M_{Z}\right)=0.1184$ [46] we find:

\begin{tabular}{|l|l|}
\hline$g_{s}\left(M_{t}\right)$ & 1.1667 \\
$g_{2}\left(M_{t}\right)$ & 0.6483 \\
$g_{1}\left(M_{t}\right)$ & 0.3587 \\
$y_{t}\left(M_{t}\right)$ & $0.93543 \pm 0.00050_{\text {(th,match) }}$ \\
$\lambda\left(M_{t}\right)$ & $0.12761 \pm 0.00030_{\text {(th,match) }}$ \\
\hline
\end{tabular}

Table 1: Values for the SM couplings in the $\overline{\mathrm{MS}}$-scheme at $\mu=M_{t}$ with the theoretical uncertainties for $y_{t}$ and $\lambda$ stemming from the matching procedure [8].

\section{The evolution of $\lambda$}

Using the three-loop $\beta$-functions for $\lambda, y_{t}, g_{s}, g_{2}$ and $g_{1}$ as well as the initial conditions from Tab. 2 we can plot the evolution of $\lambda$ up to the Planck scale $M_{\text {Planck }} \sim 10^{18} \mathrm{GeV}$. For the experimental input parameters $M_{t}=173.07 \mathrm{GeV}, M_{H}=125.9 \mathrm{GeV}$ and $\alpha_{s}\left(M_{Z}\right)=0.1184$ we find the

\footnotetext{
${ }^{2}$ There is also an upper bound $m_{\max }$ on the Higgs mass stemming from the requirement that no Landau pole appears at energies $\mu \leq \Lambda$.

${ }^{3}$ This is particularly justified at a future linear $e^{+} e^{-}$collider, where the top mass for instance will be measured at the production threshold.
} 


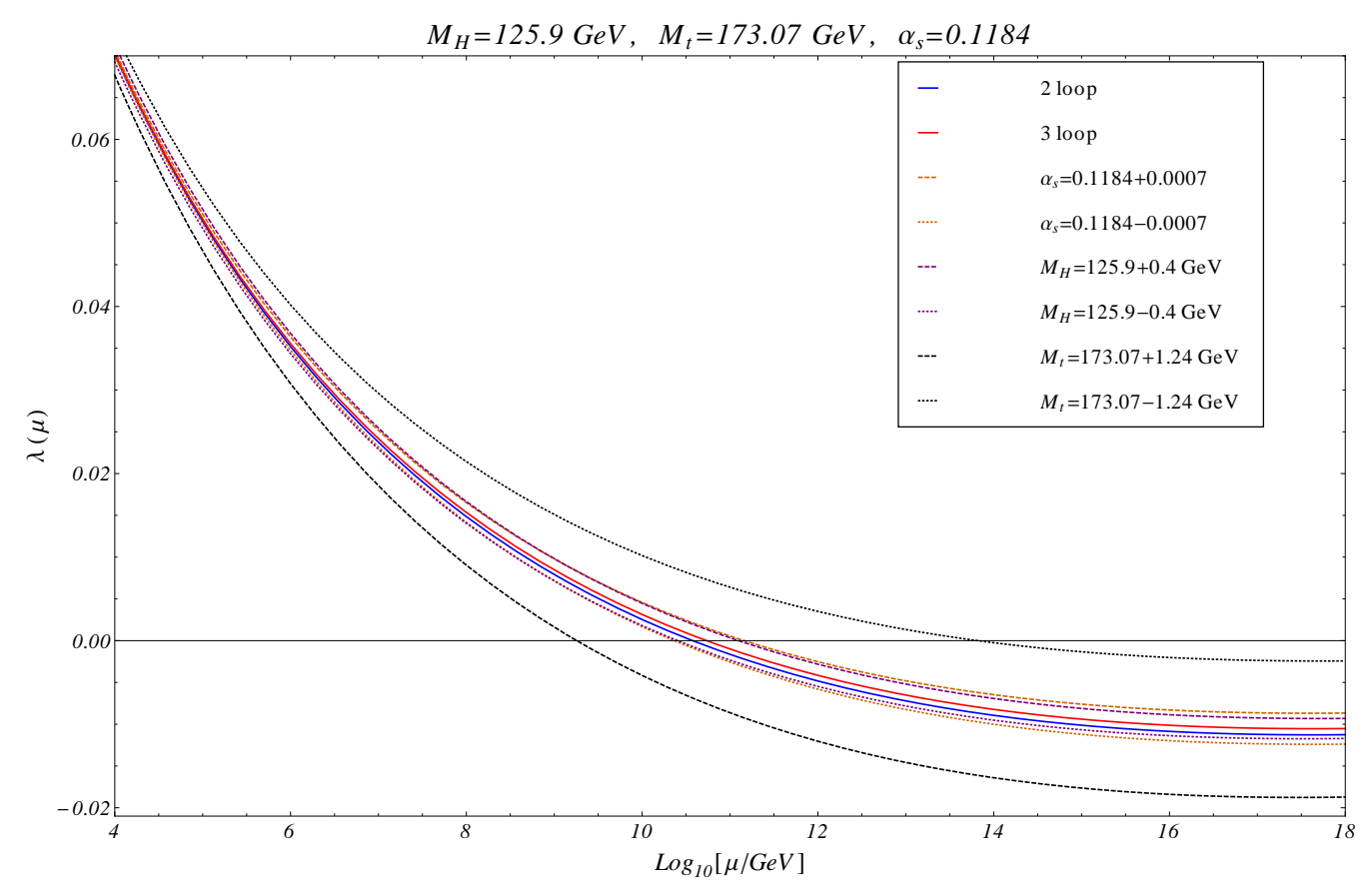

Figure 3: Evolution of $\lambda$ : experimental uncertainties

red curve in Fig. 3 which shows that $\lambda$ becomes negative at about $10^{11} \mathrm{GeV}$. If we compare this to the evolution of $\lambda$ using only two-loop $\beta$-functions for the SM couplings (blue curve), we only find a small deviation. The difference between these two curves can be interpreted as a measure for the theoretical uncertainty stemming from the truncation of the perturbation series in the calculation of $\beta$-functions. In contrast the experimental uncertainties are significantly larger. The dashed (dotted) lines show the behaviour of $\lambda$ evolved using three-loop $\beta$-functions but with $M_{t}$, $M_{H}$ and $\alpha_{s}$ increased (decreased) by one standard deviation (values from [46]). The uncertainties originating from the experimental values for $\alpha_{s}$ and $M_{H}$ are roughly of the same size and a factor 2-3 larger than the difference between the two-loop and three-loop curves at the Planck scale. In contrast, the uncertainty stemming from the top mass measurement is about an order of magnitude larger than the theoretical one at $\mu \sim 10^{18} \mathrm{GeV}$. It is worthy of note that if we shift all three experimental input parameters by one standard deviation in the more stable direction, i.e. $M_{t}=(173.07-1.24) \mathrm{GeV}, M_{H}=(125.9+0.4) \mathrm{GeV}$ and $\alpha_{s}\left(M_{Z}\right)=(0.1184+0.0007)$, we find $\lambda\left(\mu=10^{18} \mathrm{GeV}\right) \approx 0.00065>0$ and hence a stable vacuum up to the Planck scale.

It is interesting to compare these uncertainties to the ones introduced by the matching procedure (see Table 1), i.e. by truncating the perturbation series in the matching formulas. In Fig. 4 the experimental input parameters are fixed to $M_{t}=173.07 \mathrm{GeV}, M_{H}=125.9 \mathrm{GeV}$ and $\alpha_{s}\left(M_{Z}\right)=0.1184$ and the $\lambda$-curves derived from two-loop (blue) and three-loop (red) $\beta$-functions are given again. We focus on the region $\mu=10^{16}$ to $10^{18} \mathrm{GeV}$ where the distances between different lines are largest. The purple curves mark the uncertainty band due to the matching of the on-shell parameters to $\lambda$ in the $\overline{\mathrm{MS}}$-scheme and the black curves describe the uncertainty originating from the matching to $y_{t}$ in the $\overline{\mathrm{MS}}$-scheme. This plot clearly shows that all theoretical errors 
are roughly of the same size and considerably smaller than the experimental ones.

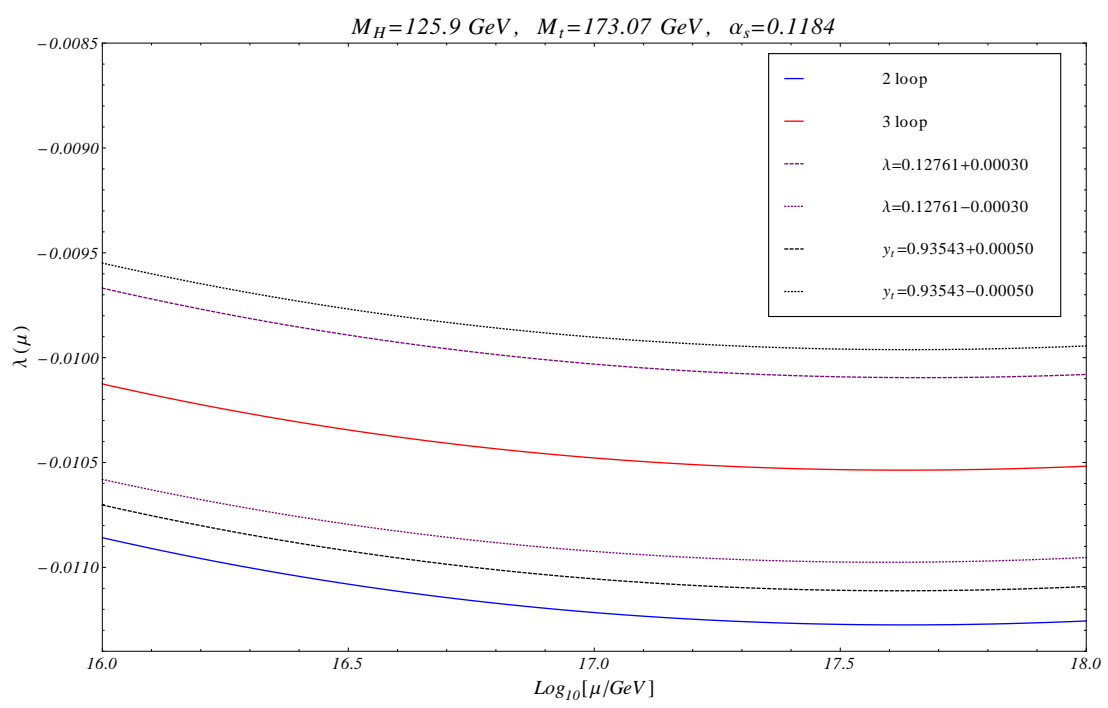

Figure 4: Evolution of $\lambda$ : matching uncertainties

\section{Conclusion}

The stability of the electroweak vacuum state is an interesting and fundamental problem in the SM framework. Although it looks as if - in the absence of new physics - a metastable scenario is the most likely, the present uncertainties do not allow for a definitive answer. The analysis presented in this talk shows that the theoretical uncertainties are well under control due to the calculation of three-loop $\beta$-functions for the Higgs self-interaction and all other SM couplings as well as due to improved precision in the matching relations between on-shell and $\overline{\mathrm{MS}}$-parameters. A more precise measurement of the experimental input parameters, especially the top mass, will hopefully lead to a clarification of this issue in the future.

\section{Acknowledgments}

I thank my collaborator K. G. Chetyrkin for invaluable discussions and J. Kühn for his support and useful comments. This work has been supported by the Deutsche Forschungsgemeinschaft in the Sonderforschungsbereich/Transregio SFB/TR-9 "Computational Particle Physics" and the Graduiertenkolleg "Elementarteilchenphysik bei höchsten Energien und höchster Präzission"

\section{References}

[1] ATLAS Collaboration, G. Aad et al., Phys. Lett. B710 (2012) 49-66, arXiv: 1202.1408 .

[2] CMS Collaboration, S. Chatrchyan et al., Phys. Lett. B 710, 26 (2012), arXiv: 1202.1488 .

[3] S. Coleman and E. Weinberg, Phys. Rev. D 7 (1973) 1888-1910. 
[4] F. Bezrukov, M. Y. Kalmykov, B. A. Kniehl, and M. Shaposhnikov, JHEP 1210, 140 (2012), arXiv: 1205.2893.

[5] G. Altarelli and G. Isidori, Physics Letters B 337 (1994) no. 1-2, 141-144.

[6] N. Cabibbo, L. Maiani, G. Parisi, and R. Petronzio, Nucl. Phys. B158 (1979) 295-305.

[7] C. Ford, D. Jones, P. Stephenson, and M. Einhorn, Nucl. Phys. B395 (1993) 17-34, arXiv: hep-lat/9210033 .

[8] D. Buttazzo, G. Degrassi, P. P. Giardino, G. F. Giudice, F. Sala, et al., arXiv:1307.3536 .

[9] I. Masina, Phys. Rev. D 87, no. 5, 053001 (2013), arXiv:1209. 0393.

[10] G. Degrassi, S. Di Vita, J. Elias-Miro, J. R. Espinosa, G. F. Giudice, et al., JHEP 1208 (2012) 098, arXiv: 1205.6497.

[11] M. Zoller, arXiv:1209.5609.

[12] K. Chetyrkin and M. Zoller, JHEP 1206 (2012) 033, arXiv: 1205.2892 .

[13] J. Elias-Miro, J. R. Espinosa, G. F. Giudice, G. Isidori, A. Riotto, et al., Phys. Lett. B709 (2012) 222-228, arXiv: 1112.3022 .

[14] M. Holthausen, K. S. Lim, and M. Lindner, JHEP 1202 (2012) 037, arXiv: 1112.2415.

[15] K. Chetyrkin and M. Zoller, JHEP 1304 (2013) 091, arXiv: 1303.2890 .

[16] A. Bednyakov, A. Pikelner, and V. Velizhanin, Nucl. Phys. B 875, 552 (2013), arXiv: 1303.4364.

[17] L. N. Mihaila, J. Salomon, and M. Steinhauser, Phys. Rev. Lett. 108 (2012) 151602.

[18] L. N. Mihaila, J. Salomon, and M. Steinhauser, Phys. Rev. D 86, 096008 (2012), arXiv: 1208 . 3357 .

[19] A. Bednyakov, A. Pikelner, and V. Velizhanin, JHEP 1301 (2013) 017, arXiv: 1210.6873.

[20] A. Bednyakov, A. Pikelner, and V. Velizhanin, Phys. Lett. B 722, 336 (2013), arXiv: 1212 . 6829.

[21] D. J. Gross and F. Wilczek, Phys. Rev. Lett. 30 (1973) 1343-1346.

[22] H. D. Politzer, Phys. Rev. Lett. 30 (1973) 1346-1349.

[23] D. Jones, Nuclear Physics B 75 (1974) no. 3, 531-538.

[24] O. Tarasov and A. Vladimirov, Sov.J.Nucl.Phys. 25 (1977) 585.

[25] W. E. Caswell, Phys.Rev.Lett. 33 (1974) 244-246.

[26] E. Egorian and O. Tarasov, Teor.Mat.Fiz. 41 (1979) 26-32.

[27] D. R. T. Jones, Phys. Rev. D 25 (1982) 581-582.

[28] M. S. Fischler and C. T. Hill, Nucl.Phys. B193 (1981) 53.

[29] M. Fischler and J. Oliensis, Phys. Lett. B 119 (1982) no. 4, 385-386.

[30] I. Jack and H. Osborn, Nucl. Phys. B 249 (1985) no. 3, 472-506.

[31] M. E. Machacek and M. T. Vaughn, Nucl. Phys. B 222 (1983) no. 1, 83-103.

[32] M. E. Machacek and M. T. Vaughn, Nucl. Phys. B 236 (1984) no. 1, 221-232.

[33] M. E. Machacek and M. T. Vaughn, Nucl. Phys. B 249 (1985) no. 1, 70-92.

[34] M.-x. Luo and Y. Xiao, Phys. Rev. Lett. 90 (2003) 011601, arXiv: hep-ph/ 0207271 .

[35] C. Ford, I. Jack, and D. Jones,Nucl.Phys. B387 (1992) 373-390, arXiv: hep-ph/ 0111190.

[36] T. Curtright, Phys.Rev. D21 (1980) 1543.

[37] D. Jones, Phys.Rev. D22 (1980) 3140-3141.

[38] O. Tarasov, A. Vladimirov, and A. Y. Zharkov, Phys.Lett. B93 (1980) 429-432.

[39] S. Larin and J. Vermaseren, Phys. Lett. B303 (1993) 334-336, arXiv: hep-ph/9302208 .

[40] M. Steinhauser, Phys.Rev. D59 (1999) 054005, arXiv: hep-ph/9809507 .

[41] A. Pickering, J. Gracey, and D. Jones, Phys.Lett. B510 (2001) 347-354, arXiv: hep-ph 0104247.

[42] F. Jegerlehner, M. Y. Kalmykov, and B. A. Kniehl, Phys.Lett. B722 (2013) 123-129, arXiv:1212 . 4319.

[43] J. Espinosa, G. Giudice, and A. Riotto, JCAP 0805 (2008) 002, arXiv : 0710.2484.

[44] R. Hempfling and B. A. Kniehl, Phys.Rev. D51 (1995) 1386-1394, arXiv: hep-ph/9408313 .

[45] A. Sirlin and R. Zucchini, Nucl. Phys. B 266 (1986) no. 2, 389-409.

[46] K. Nakamura et al., J. Phys. G (2010) no. 37, 075021. 